\title{
Desarrollo de Competencias Transversales en la Universidad de Murcia: Fortalezas, Debilidades y Propuestas de Mejora
}

\author{
Development of Cross-sectional Competences at Universidad de Murcia: \\ Strengths, Weaknesses, and Proposals for Improvement \\ Desenvolvimento de Competências Transversais na Universidade de Múrcia: \\ Fortalezas, Fraquezas e Propostas de Melhora
}

\section{Natalia González Morga* https://orcid.org/0000-0002-5544-4464}

Departamento de Métodos de Investigación y Diagnóstico en Educación, Facultad de Educación, Universidad de Murcia, España

Javier Pérez Cusó** https://orcid.org/0000-0003-1376-1776

Departamento de Métodos de Investigación y Diagnóstico en Educación, Facultad de Educación, Universidad de Murcia, España.

Mirian Martínez Juárez ${ }^{* * *}$ https://orcid.org/0000-0002-9618-0194

Departamento de Métodos de Investigación y Diagnóstico en Educación, Facultad de Educación, Universidad de Murcia, España

$\nabla$

Recibido: 27-03-18 Revisado: 10-06-18 Aceptado: 10-10-18 Publicado: 20-12-18

- Resumen. El desarrollo de competencias transversales en la educación superior es de especial interés por su vinculación al contexto socio-laboral. La inclusión de estas competencias en el currículo universitario es irregular y compleja. El trabajo en equipo, interacción social, compromiso ético-social y control emocional son, entre otras, competencias clave de los profesionales de nuestro tiempo. Desde este trabajo se pretende conocer el grado de desarrollo de estas cuatro competencias transversales y
Palabras clave:

Competencias

transversales, educación

superior, mejora 
se identifican las fortalezas y debilidades en su proceso de formación, para finalmente analizar las propuestas de mejora que aportan 1137 estudiantes de último año de Grado de las cinco ramas de conocimiento de la Universidad de Murcia. Se aplica el cuestionario CECTGRA, cuyas respuestas se analizan desde un enfoque mixto que alterna el uso del programa SPSS v23 y ATLAS.TI 7. Los resultados indican la necesidad de mejorar el desarrollo de las competencias; el trabajo en equipo es la más desarrollada, encontrándose en el polo opuesto, el control emocional, hallándose diferencias significativas entre las ramas de conocimiento. Las fortalezas identificadas se agrupan en relación con el propio estudiante, el entorno y la estructura, y las debilidades y propuestas de mejora en torno a la institución, el centro y el aula.

- Abstract. The development of cross-sectional competences in higher education is of special interest because of its link to the socio-labor context. The inclusion of these competences in the university curriculum is irregular and complex. Teamwork, social interaction, ethical-social commitment, and emotional control are, among others, key competences of professionals nowadays. The aim of this work is to ascertain the degree of development of these four cross-sectional competences and to identify the strengths and weaknesses in their training process, and finally to analyze the proposals for improvement provided by 1,137 students in their final year of undergraduate studies from the five branches of knowledge at Universidad de Murcia. The CECTGRA questionnaire was applied and its answers were analyzed from a mixed approach alternating the use of the SPSS v23 and ATLAS.TI 7 programs. The results point out towards the need to improve the development of competences; teamwork is the most developed, with emotional control at the opposite end, with significant differences among each branch of knowledge. The strengths identified are grouped in relation to students themselves, the environment, and the structure; and the weaknesses and proposals for improvement revolving on the institution, the center, and the classroom.

- Resumo. O desenvolvimento de competências transversais na educação superior é de especial interesse por sua vinculação com o contexto social e do trabalho. A inclusão destas competências na grade curricular universitária é irregular e complexa. O trabalho em equipe, interação social, compromisso ético-social e o controle emocional são, dentre outras, competências chave dos profissionais de nossos tempos. Desde este trabalho pretende-se conhecer o grau de desenvolvimento destas quatro competências transversais e identificam-se as fortalezas e fraquezas em seu processo de formação, para de la educación, estudiante.

\section{Keywords: \\ cross-sectional competences, higher education, improvement of education, student}
Palavras-chave: Competências transversais, educação superior, melhora da educação, estudante. 
finalmente analisar as propostas de melhora por parte de 1137 estudantes do último ano de Grau das cinco áreas de conhecimento da Universidade de Múrcia. Aplica-se o questionário CECTGRA, cujas respostas são analisadas desde uma abordagem mista que alterna o uso do programa SPSS v23 e ATLAS.TI 7. Os resultados apontam para a necessidade de melhorar o desenvolvimento das competências; salientam que a competência de trabalho em equipe é a mais desenvolvida, e destacam que no polo oposto está o controle emocional. Além disso, encontram-se diferenças significativas entre as áreas de conhecimento. As fortalezas identificadas agrupam-se em relação com o próprio estudante, o entorno e a estrutura, e as fraquezas e propostas de melhora com relação à instituição, o centro e a aula.

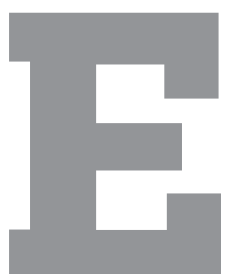

1 desarrollo de las competencias transversales en la Educación Superior supone un importante ámbito de discusión y preocupación de las agencias y organismos internacionales (CEDEFOP, 2014; OCDE, 2016; OIT, 2015; Unesco, 2016). Señalan la importancia de profundizar en la formación de los futuros egresados y en la necesidad de mejorar el ajuste entre sus competencias y los requerimientos de un mercado de trabajo que cada vez precisa un mayor nivel de flexibilidad y capacidad de adaptar los conocimientos al contexto concreto de trabajo y las necesidades y problemas que este plantea.

Este interés por el desarrollo de competencias transversales en el ámbito universitario se ha visto reflejado en multitud de estudios (Aguado, González, Antúnez \& de Dios, 2017; Jato, Cajide, Muñoz \& García, 2016; Mareque \& De Prada, 2018; Martínez Clares \& González Morga, 2018a; Robledo, Fidalgo, Arias \& Álvarez, 2015; Rodríguez, Ibarra \& Cubero, 2018; Solanes, Núñez $\&$ Rodríguez, 2008) que abordan desde diferentes perspectivas el desarrollo e importancia de las competencias transversales en el contexto universitario.

El conocimiento se ha convertido en un factor clave en el desarrollo productivo, económico, social y laboral, por lo que el modo en que lo gestionamos tiene un impacto directo en todos los ámbitos y esferas vitales. En este contexto, las Tecnologías de la Información y la Comunicación (TIC) han creado un sustrato que obliga a poner el foco en cómo se gestiona, transmite y difunde la información y el conocimiento, siendo la sociedad en su conjunto responsable de estos procesos, pero muy especialmente la Educación Superior que, como señalan Hargreaves y Fullan (2014), se constituye como la institución de referencia para el avance de lo que denominan capital profesional en nuestra sociedad. Como señalan estos autores este capital profesional no se compone únicamente de los conocimientos propios del ámbito profesional sino también de lo que denominan capital social y decisorio. Desde esta óptica la Educación Superior ha de ir 
más allá de la transmisión de contenidos teórico-prácticos dentro de una disciplina profesional y abordar la transmisión de otro tipo de saberes que conforman dicho capital.

En este mismo sentido hay un acuerdo generalizado sobre la necesidad de profundizar en el desarrollo de las competencias transversales por parte del alumnado universitario, dado que este desarrollo es clave para alcanzar la necesaria coherencia entre las demandas actuales de los empleadores y la formación de los egresados de la Universidad del siglo XXI (Mareque \& De Prada, 2018; OIT, 2015; Teichler, 2015).

Por ello se ha planteado en la última década una transformación estructural en las metodologías de enseñanza en la universidad hacia un enfoque más centrado en el aprendizaje del alumnado: metodologías activas, aprendizaje por proyectos, resolución de problemas o tutorización del alumnado como medio para la personalización del aprendizaje, que permitan el desarrollo de competencias en los futuros egresados (Martínez Clares \& González Morga, 2018b). Pero, como destaca Ursin (2017), los cambios estructurales y normativos tardan en llegar a las aulas. Pese a un amplio consenso normativo y social en torno a la necesidad de modificar en profundidad cómo se enseña y, sobre todo, cómo y qué se aprende en las aulas universitarias, todavía queda un importante camino por recorrer en este sentido.

El consenso en torno a la importancia de la introducción de las competencias en la Educación Superior implica reflexionar y modificar modos de hacer en torno a cómo se enseña, evalúa y gestionan los currículos universitarios. Aunque también puede discutirse el hecho de que la universidad se centre excesivamente en las necesidades del mercado laboral alejándose del propósito de generar conocimiento válido en sí mismo, se relacione o no con necesidades y demandas de los empleadores. Frente a este argumento, otras voces señalan que la adquisición de competencias por parte de los universitarios también contribuye a su desarrollo integral, no solo en la dimensión profesional o de inserción laboral, sino a su dimensión personal y social (Ibáñez, 2009; López Gómez, 2016).

Como señala el estudio de Rodríguez (2014), el alumnado muestra algunas reticencias al modelo de formación por competencias, puesto que le va a exigir más trabajo, aunque según estos estudios finalmente se sienten más satisfechos con los aprendizajes que consiguen.

Este discurso sobre la introducción del modelo de competencias en la Educación Superior tiene un impacto directo en cuatro grandes áreas (Manzanares \& Sánchez, 2012):

- El propio debate sobre Educación Superior, que precisa de una reflexión sobre la necesidad de aprendizaje a lo largo de la vida, la labor de la propia institución universitaria o la formación de profesionales.

- La organización y estructura de las titulaciones universitarias, dividida a partir del Proceso de Bolonia en dos ciclos diferenciados: enseñanzas de grado y enseñanzas de posgrado (máster y doctorado).

- El propio currículo, la introducción de las competencias en el diseño de las asignaturas 
(estructuradas en torno a materias) o la elaboración de un trabajo final que ponga de relieve las competencias adquiridas a lo largo de los estudios.

- La dimensión metodológica, ya que el discurso sobre competencias obliga a reenfocar la metodología empleada en el aula universitaria, pasando de estar centrada en la mera transmisión de contenidos teóricos a permitir y potenciar la adquisición de competencias.

Así, como señalan Rodríguez et al. (2018), es necesario incrementar el esfuerzo de la Educación Superior por mejorar las competencias relacionadas con la autonomía del estudiante y su capacidad reflexiva, muy relacionadas con la capacidad del aprendizaje permanente.

Pese a diferentes conceptualizaciones e, incluso, denominaciones en torno a las competencias transversales, en diferentes contextos anglosajones se refieren a ellas con diferentes adjetivos (key skills, core skills, essential skills, soft skill, employability skills, etc.) que más allá de algunos matices (Rodríguez, 2015; Unesco, 2016) hacen referencia a determinados conocimientos, actitudes y habilidades esenciales en la formación integral y en el desarrollo de la empleabilidad.

Pese a que se ha tratado en diversas ocasiones de sistematizar y clasificar las competencias transversales (Aubert \& Gilbert, 2003; Clemente \& Escribá, 2013; González \& González, 2009), la tipología más aceptada en el ámbito educativo es la sugerida desde el proyecto Tuning, que presenta un modelo de tres dimensiones: competencias instrumentales, aquellas que van a permitir el desarrollo académico del estudiante, están ligadas a capacidades y habilidades de carácter instrumental; competencias interpersonales, relacionadas con las habilidades para la relación con los demás, el trabajo en equipo y, en general, inciden en facilitar la interacción, colaboración y cooperación; competencias sistémicas, que requieren el manejo previo de las competencias instrumentales e interpersonales. Por su parte, en el trabajo de Solanes et al. (2008) se desagregan estas tres dimensiones en un total de siete:

- Instrumentales: Desempeño del trabajo y habilidades de gestión.

- Interpersonales: Relaciones interpersonales y de trabajo en equipo.

- Sistémicas: Liderazgo, motivación por el trabajo y capacidad de aprendizaje.

La complejidad de las competencias transversales lleva a que se pueda encontrar multitud de aproximaciones tanto en su denominación como en clasificaciones o tipologías. Por ello, para comprenderlas con una mayor profundidad, es necesario analizar algunas características constituyentes de las mismas (González Morga, 2017; OIT, 2015; Rychen \& Salganic, 2003).

- Tienen un carácter transversal y transferible, no se aprenden y aplican en un único contexto, sino que van a poder emplearse en diferentes dimensiones y ámbitos de la persona.

- Permiten abordar múltiples situaciones, no solo pueden aplicarse en diferentes contextos, sino que sirven para afrontar y resolver aspectos de diversa índole. 
- No son específicas de una ocupación, sino que son necesarias para desarrollar multitud de empleos en organizaciones muy distintas y a diferentes niveles jerárquicos dentro de las mismas.

- No están asociadas a contenidos específicos que puedan mutar con el paso del tiempo, sino que tienen un carácter más estructural, son estables en el tiempo.

Como señala Blanco (2009) el cambio en el enfoque metodológico exigido por la aplicación del modelo de aprendizaje de competencias en la universidad ha afectado tanto al rol del profesorado como del propio alumno. Por su parte, el profesor ha de modificar su papel, pasa de ser transmisor de conocimientos a facilitador de aprendizajes, proponiendo experiencias formativas, mientras que, por otro lado, el alumnado ha de abandonar un rol más pasivo y convertirse en un agente activo de su propio aprendizaje.

Esta exigencia a ambos agentes universitarios plantea la necesidad de revisar cómo profesores y estudiantes se acercan y perciben el modelo de competencias, cómo están jugando su papel en los procesos de enseñanza y aprendizaje. Es necesario conocer si alumnos y profesores asumen la imperiosa necesidad del modelo y, verdaderamente, están trabajando de otro modo en las aulas universitarias, además de cómo es posible mejorar esta práctica.

Por ello, se están desarrollando diferentes investigaciones en torno a la percepción del desarrollo de competencias en las universidades españolas, tanto desde la percepción del alumnado (Clemente \& Escribá, 2013; Herrero, González \& Marín, 2015; Rodríguez et al., 2018) como desde el punto de vista de los ya egresados e incluso de los empleadores (Freire, Teijeiro \& Pais, 2013; Michavila, Martínez, Martín, García \& Cruz, 2016; Palmer, Montaño \& Palou, 2009).

El estudio de Sarceda \& Rodicio (2018), sobre la formación de competencias generales, específicas y transversales, encuentra que el alumnado percibe que las primeras se abordan en mayor medida en la institución universitaria, las específicas en los centros de prácticas, mientras que las transversales se abordan en menor medida. En este mismo sentido, el estudio desarrollado por Mareque \& De Prada (2018) señala que algunas competencias transversales, como la creatividad, no se desarrollan de modo suficiente.

Algunos estudios señalan que determinadas estrategias metodológicas contribuyen en mayor medida a la consecución de algunas tipologías de competencias. Por ejemplo, el trabajo de Robledo et al. (2015) señala, en una comparación entre diferentes metodologías (Aprendizaje Basado en Problemas [ABP], método de casos, método de expertos, método de estudio compartido y método de estudio dirigido), que el ABP es más efectivo a la hora de desarrollar competencias instrumentales, sistémicas o interpersonales entre el alumnado. Como señalan los autores de este estudio, emergen dos posibles razones para la mayor eficacia de este enfoque metodológico: la vinculación entre conocimiento y práctica profesional y la exigencia de una mayor autonomía por parte del estudiante. Sin embargo, aconsejan el empleo de diferentes metodologías, de modo que se potencien y favorezcan un mayor rango de competencias. 
Queda claro que la incorporación de las competencias $\mathrm{y}$, específicamente, de las competencias transversales por parte de la universidad no ha sido un proceso sencillo, por lo que desde este trabajo se pretende contribuir a un mejor conocimiento de cómo se ha integrado la enseñanza-aprendizaje de las competencias en la formación de los estudiantes y conocer qué acciones podrían desarrollarse para la mejora de la formación en competencias.

Para ello se establecen los siguientes objetivos de investigación:

1. Conocer el desarrollo de las competencias transversales en las aulas de la Universidad de Murcia desde la percepción del alumnado de último curso de Grado.

2. Identificar las fortalezas y debilidades de la formación en competencias transversales a través de la visión del alumnado.

3. Analizar las propuestas de mejora que establece el alumnado sobre la formación en competencias transversales.

\section{MÉTODO}

\section{Diseño}

La investigación se desarrolla bajo un diseño no experimental, descriptivo y transversal. Entre los métodos o técnicas de recogida de datos se opta por la encuesta o survey y se materializa en un cuestionario que alterna preguntas cuantitativas y cualitativas, de ahí que se requiera de un enfoque analítico mixto para el análisis de los datos.

\section{Participantes}

La población objeto de estudio corresponde al alumnado de $4^{\circ}$ de Grado de la Universidad de Murcia. La selección de los participantes se realiza a través de un muestreo aleatorio estratificado con el que se obtiene una muestra total de 1137 estudiantes, representativa del alumnado de último curso de Grado de dicha universidad con un nivel de confianza de $99 \%$ y un error muestral de $3.44 \%$.

Los participantes se distribuyen en diferentes ramas de conocimiento, respetando la proporcionalidad del conjunto de los estratos en la población global. El 50\% son estudiantes de la rama de Ciencias Sociales, el 16\% de Ciencias de la Salud, el 14\% pertenece a Ciencias Experimentales, el 16\% a Artes y Humanidades y el 4\% a Ingenierías. Del total de los estudiantes que responden al cuestionario, el 68\% son mujeres y el 31\% restante hombres y la edad media de todos ellos se sitúa en 23 años (DE=4.16). 


\section{Instrumentos}

La recogida de información se realiza a partir del Cuestionario de Evaluación de Competencias Transversales de Grado (CECTGRA) diseñado ad hoc. Este cuestionario integra cinco bloques de preguntas elaborados en torno a una escala de competencias que recoge la valoración del desarrollo o grado de enseñanza de las competencias transversales en las aulas universitarias, el dominio o adquisición que presenta el estudiante y la relevancia o valor que se otorga para el desarrollo profesional.

El listado de competencias transversales que contempla la escala fue definido ad hoc por Martínez Clares y González Morga (2018a) tras el análisis de documentos clave para el diseño de los títulos de Grado y diversos estudios que contemplan la visión de los empleadores sobre las competencias más requeridas en el mercado productivo en los últimos años. Esta relación de competencias se agrupa en función del modelo Tuning, adoptado por la Agencia Nacional de Evaluación de la Calidad y Acreditación (ANECA) para la elaboración de los libros blancos de las titulaciones universitarias españolas, cuyas competencias se estructuran en instrumentales, interpersonales y sistémicas.

Su estructura interna fue validada a través de análisis factorial confirmatorio con unos indicadores de bondad de ajuste satisfactorios (dimensión desarrollo: CFI=.094; NFI=938; RMSEA=.072) y comprobada su fiabilidad tanto de la escala global (alfa=.927) como de las subescalas resultantes (competencias instrumentales, alfa=.785; competencias interpersonales, alfa=.825; competencias sistémicas, alfa=.899).

Para dar respuesta al objetivo de este trabajo, se presentan los resultados obtenidos en el análisis de la dimensión desarrollo de las competencias interpersonales (trabajo en equipo, interacción social, compromiso ético y social, y control emocional) y las preguntas abiertas relativas a puntos fuertes y débiles de la formación universitaria en competencias transversales, así como en las propuestas para su mejora.

\section{Procedimiento}

Una vez diseñado el cuestionario y sometido a un proceso de validación de contenido a través de juicio de expertos y el método de agregados individuales, se realiza la recogida de información. Esta se planifica en el último año de Grado con la intención de que el alumnado tuviera una percepción lo más completa posible de la formación universitaria. Para la aplicación del cuestionario, se contacta con los tutores de cada grupo-clase y son los propios miembros del equipo de investigación quienes se trasladan al aula para realizar una breve explicación del cuestionario e insistir en su carácter voluntario, anónimo y confidencial.

Para el análisis de los datos cuantitativos se recurre tanto a la estadística descriptiva (media, desviación típica) como a la inferencial, a través de pruebas no paramétricas (Friedman para 
muestras relacionadas, U de Mann Whitney para dos muestras independientes y H de Kruskal Wallis para muestras independientes de más de dos grupos), dada la no distribución normal de los datos probada con la prueba K-S. Para el tratamiento de los datos cuantitativos, se usa el software estadístico SPSS v.23. El análisis de los datos cualitativos se realiza a través de un procedimiento de reducción de la información, categorización e interpretación de resultados mediante redes semánticas o network. Para ello se utiliza como soporte o herramienta para clasificar, seleccionar y representar la información, el programa ATLAS.ti 7.

\section{Resultados}

El desarrollo de las competencias transversales en las aulas universitarias se considera aceptable, si bien bastante mejorable con una media en torno al valor de 3. La competencia más desarrollada, según la percepción del estudiante, corresponde al trabajo en equipo, mientras que la menos desarrollada es el control emocional, tal y como indican los estadísticos de la Tabla 1.

\section{Tabla 1}

Análisis descriptivo e inferencial del desarrollo de las competencias transversales

\begin{tabular}{|cccc|}
\hline COMPETENCIA & $\mathbf{M}$ & DE & $\begin{array}{c}\text { PRUEBA DE FRIEDMAN } \\
\text { CHI-CUADRADO } \\
\text { (GL) } \\
\text { SIG. }\end{array}$ \\
\hline Trabajo en equipo & 3.54 & 0.94 & 571.227 \\
Interacción social & 3.12 & 1.04 & $(3)$ \\
Compromiso ético y social & 3.51 & 0.98 & $<.001$ \\
Control emocional & 2.88 & 1.13 & \\
\hline
\end{tabular}

En un análisis más detallado de las unidades de competencia que integra esta dimensión competencial, el mayor desarrollo se percibe en la competencia relacionada con la valoración y respeto a la diversidad y multiculturalidad $(\mathrm{X}=3.86)$, seguido muy de cerca del trabajo colaborativo y cooperativo en equipos multidisciplinares $(\mathrm{X}=3.85)$.

Por el contrario, aquella unidad competencial menos trabajada durante los años de carrera corresponde a la tolerancia a la frustración y la adversidad ( $\mathrm{X}=2.66)$ seguido muy de cerca del manejo del estrés (X=2.67). Estos resultados se aprecian pormenorizadamente en la Figura 1. 


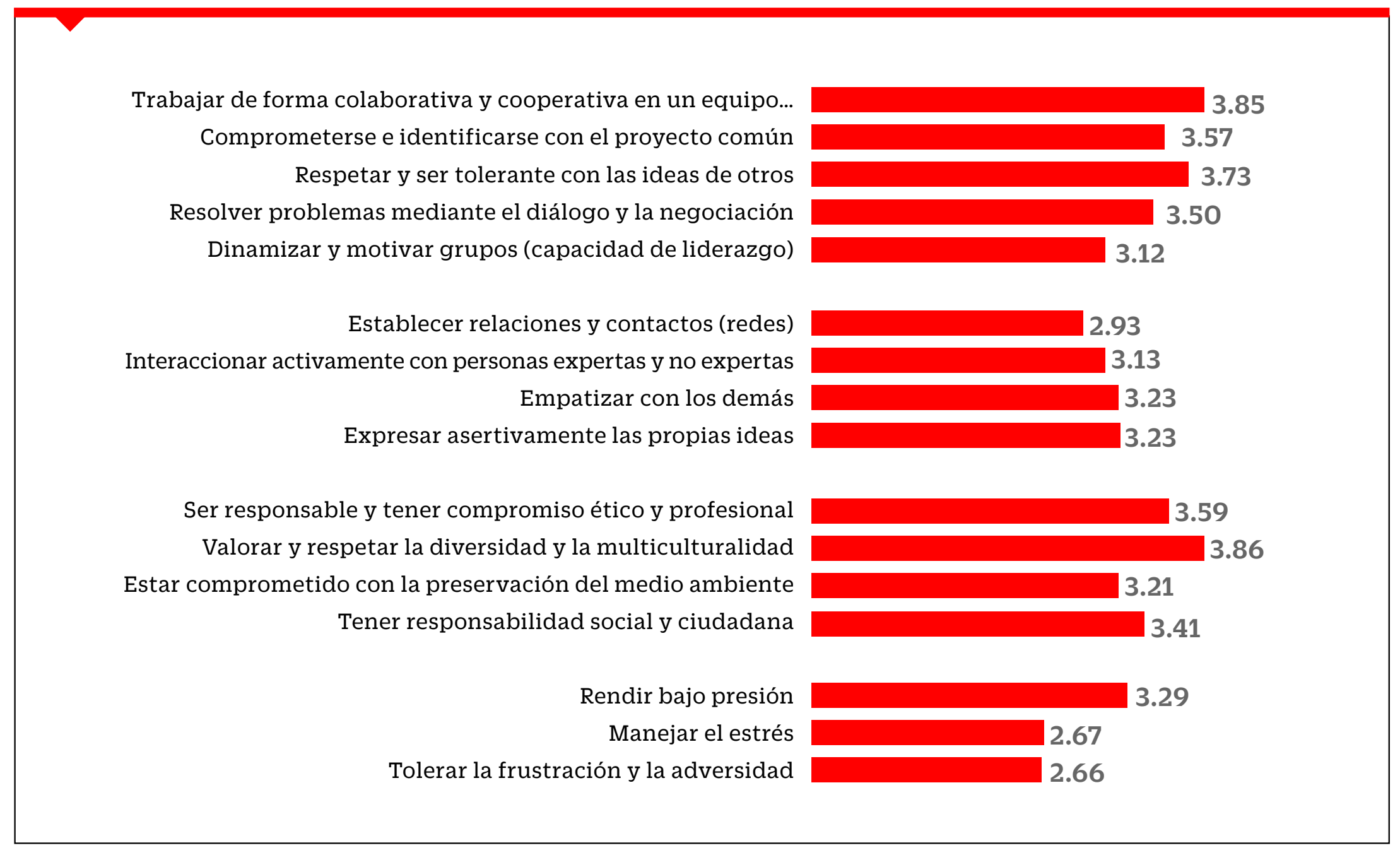

Figura 1. Análisis descriptivo del desarrollo de las unidades que integran las competencias transversales.

El desarrollo competencial además difiere de una rama de conocimiento a otra de forma significativa $(p<.015)$ y en función del sexo de los estudiantes $(p=.000)$. Estos resultados muestran que son los estudiantes de Ciencias Sociales los que perciben el desarrollo más elevado de la competencia de trabajo en equipo en contraposición con los estudiantes de Ciencias Experimentales, que se atribuyen el grado de desarrollo más bajo. Por otro lado, en la competencia de interacción social destacan los estudiantes de Ciencias de la Salud con el desarrollo más elevado durante su formación, mientras los estudiantes de Ingeniería obtienen la puntuación más baja. De igual forma ocurre con la competencia de compromiso ético social, pero en este caso los estudiantes de Ciencias Sociales vuelven a destacar por el desarrollo más alto. Finalmente son los estudiantes de Ingeniería los que perciben un desarrollo más elevado de la competencia de control emocional $(\mathrm{X}=3.37)$ frente al resto de titulaciones que muestran un valor medio que no supera el 3 en ningún caso y muy próximos entre sí (ver Tabla 2). 


\section{Tabla 2}

Análisis descriptivo e inferencial del desarrollo de las competencias transversales en función de la rama de conocimiento

\begin{tabular}{|c|c|c|c|c|}
\hline COMPETENCIA & RAMA DE CONOCIMIENTO & $\mathbf{M}$ & $\mathrm{DE}$ & $\begin{array}{c}\text { CHI-CUADRADO } \\
\text { (GL) } \\
\text { SIG. }\end{array}$ \\
\hline \multirow{6}{*}{ Trabajar en equipo } & & 3.78 & 0.88 & \multirow{6}{*}{$\begin{array}{c}82.443 \\
(4) \\
<.001\end{array}$} \\
\hline & Ciencias Sociales & & & \\
\hline & Ciencias de la Salud & 3.49 & 0.85 & \\
\hline & Ciencias Experimentales & 3.17 & 0.94 & \\
\hline & Artes y Humanidades & 3.27 & 1.01 & \\
\hline & Ingeniería & 3.27 & 0.89 & \\
\hline \multirow{5}{*}{ Interacción social } & Ciencias Sociales & 3.27 & 0.99 & \multirow{5}{*}{$\begin{array}{c}75.987 \\
(4) \\
<.001\end{array}$} \\
\hline & Ciencias de la Salud & 3.41 & 0.95 & \\
\hline & Ciencias Experimentales & 2.66 & 1.03 & \\
\hline & Artes y Humanidades & 2.90 & 1.10 & \\
\hline & Ingeniería & 2.63 & 0.88 & \\
\hline \multirow{5}{*}{$\begin{array}{l}\text { Compromiso ético } \\
\text { y social }\end{array}$} & Ciencias Sociales & 3.61 & 0.93 & \multirow{5}{*}{$\begin{array}{c}30.508 \\
(4) \\
<.001\end{array}$} \\
\hline & Ciencias de la Salud & 3.57 & 0.90 & \\
\hline & Ciencias Experimentales & 3.53 & 1.05 & \\
\hline & Artes y Humanidades & 3.29 & 1.06 & \\
\hline & Ingeniería & 2.93 & 1.03 & \\
\hline \multirow{5}{*}{ Control emocional } & Ciencias Sociales & 2.80 & 1.10 & \multirow{5}{*}{$\begin{array}{c}12.426 \\
(4) \\
.014\end{array}$} \\
\hline & Ciencias de la Salud & 2.98 & 1.05 & \\
\hline & Ciencias Experimentales & 2.87 & 1.19 & \\
\hline & Artes y Humanidades & 2.89 & 1.14 & \\
\hline & Ingeniería & 3.37 & 1.28 & \\
\hline
\end{tabular}

Con relación al sexo de los participantes son las mujeres las que perciben un desarrollo superior en todas las competencias $(p=.000)$, a excepción del control emocional, donde no se han encontrado diferencias estadísticamente significativas entre sexos ( $p<.132$ ). En la Tabla 3 , se aprecian los estadísticos obtenidos. 
Tabla 3

Análisis descriptivo e inferencial del desarrollo de las competencias transversales en función de la rama de conocimiento

\begin{tabular}{|lcccc|}
\hline COMPETENCIA & SEXO & M & DE & $\begin{array}{c}\text { U MANN WHITNEY } \\
\text { SIG (P). }\end{array}$ \\
\hline \multirow{2}{*}{ Trabajar en equipo } & Hombre & 3.35 & .91 & $<.001$ \\
& Mujer & 3.63 & .93 & \\
Interacción social & Hombre & 2.95 & 1.03 & $<.001$ \\
& Mujer & 3.19 & 1.03 & \\
Compromiso ético & Hombre & 3.32 & .99 & $<.001$ \\
y social & Mujer & 3.60 & 0.96 & .132 \\
Control emocional & Hombre & 2.96 & 1.15 & \\
\hline
\end{tabular}

\section{Fortalezas y debilidades de la formación en competencias transversales}

Una vez analizado el desarrollo de las competencias transversales, se identifican las fortalezas y debilidades de este proceso. En primer lugar, se determinan los aspectos principales que favorecen la formación en competencias transversales en las aulas universitarias. En la Figura 2 se presenta la red de relaciones entre las unidades de análisis construidas a partir de la opinión de los propios estudiantes.

Los códigos se agrupan en tres categorías o familias conceptuales: por un lado, el propio estudiante con su esfuerzo, interés personal y el trabajo autodidacta ${ }^{1}$, además de las capacidades propias referidas al bagaje de competencia que tienen adquiridas antes de su incorporación a la universidad, proporcionada por distintos ámbitos sociales e instituciones en los que interactúa.

Otro aspecto relevante es la realización de actividades extracurriculares por voluntad propia como cursos de formación, participar en becas de movilidad o realizar algún tipo de voluntariado. Todas ellas son actividades que fortalecen la preparación del estudiante en competencias transversales.

Por otro lado, y a consecuencia de algunas de estas actividades, se encuentran las experiencias que adquiere el estudiante en relación con su entorno (subcategoría 2) tanto las ocurridas en el aula (experiencias con sus iguales) como las proporcionadas por los programas de movilidad que facilitan estudiar en otros países o las prácticas en el extranjero (experiencias internacionales). A estas vivencias hay que añadir las procedentes del ámbito laboral. 


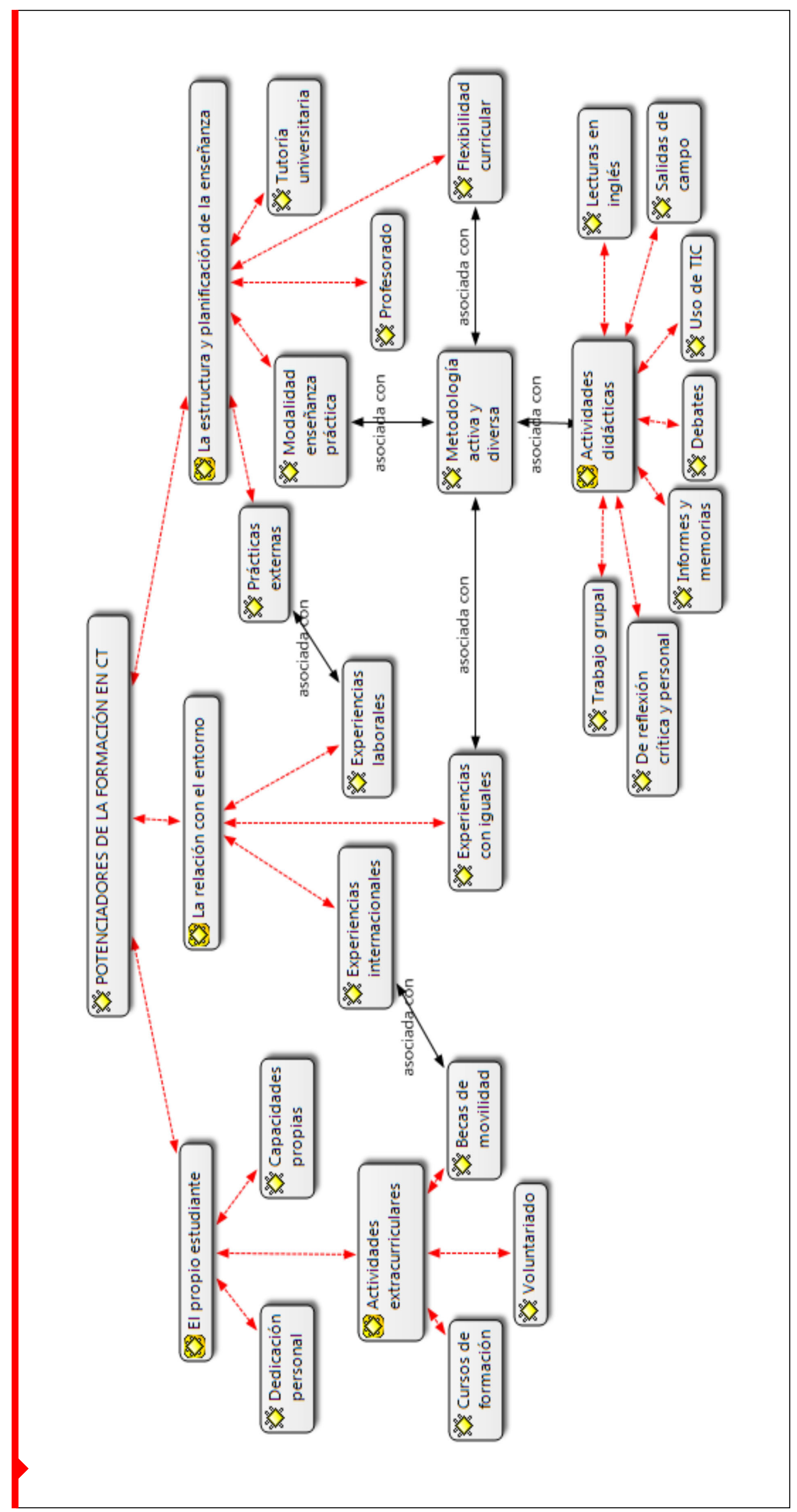

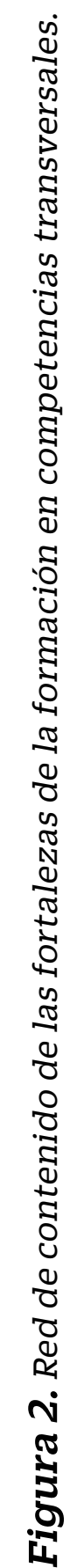


En este caso, procedentes de la combinación de estudios con trabajo, pero fundamentalmente de la realización de prácticas externas.

Las prácticas curriculares son una de las materias que conforman el plan de estudios de la mayoría de las titulaciones analizadas, clasificada dentro de la subcategoría tercera (estructura y planificación de la enseñanza) con función de nexo entre las subcategorías 2 y 3. Los estudiantes del área de Ciencias Sociales manifiestan que en las prácticas escolares aprendes y tienes vivencias que jamás se dan en la universidad, además permiten aprender la futura profesión de una manera directa.

Junto a esta materia, los estudiantes destacan la modalidad práctica de enseñanza para desarrollar las competencias transversales en el aula, manifiestan su adecuación para aprender a relacionar la teoría con la práctica. Para ello, consideran apropiado el uso de metodologías activas por su capacidad de favorecer la participación del alumnado e interacción entre compañeros, en palabras de los propios estudiantes, de generar clases dinámicas y practicar en conjunto con los compañeros; además de la diversidad de estrategias metodológicas que favorecen, por un lado, aprehender el currículo desde distintas perspectivas e interpretaciones, que a su vez permiten extrapolar los contenidos a distintas situaciones, y, por otro, atender a la diversidad de estilos de aprendizaje. De ahí que este código esté asociado a la flexibilidad curricular que percibe el estudiante para atender a sus individualidades y a la variedad de actividades y ejercicios que observan los estudiantes de las distintas ramas de conocimiento como potenciadores de la formación basada en competencias transversales. Concretamente son siete las actividades didácticas que perciben como las más adecuadas para el desarrollo de estas competencias: trabajo en equipo, exposiciones orales, ejercicios apoyados en las TIC (Videos), lecturas en inglés, debates, elaboración de informes y memorias, salidas de campo (excursiones y visitas) y ejercicios de reflexión crítica y personal.

Por último, los estudiantes también consideran las sesiones de tutoría, tanto individual como grupal, como un espacio que favorece la comunicación entre profesor y alumno, acorta distancias entre ellos y potencia dicha formación.

En relación con los aspectos que según el estudiante dificultan la formación en competencias transversales se pueden agrupar en tres subcategorías (ver Figura 3).

La primera de ellas integra obstaculizadores a nivel institucional como la escasa financiación de las universidades para implantar las reformas de Bolonia debido a la situación actual de crisis. La segunda agrupa limitaciones de la formación a nivel de centro que aluden tanto a la estructura del plan de estudios de los títulos de Grado como al enfoque de enseñanza generalizado en estos programas educativos. 


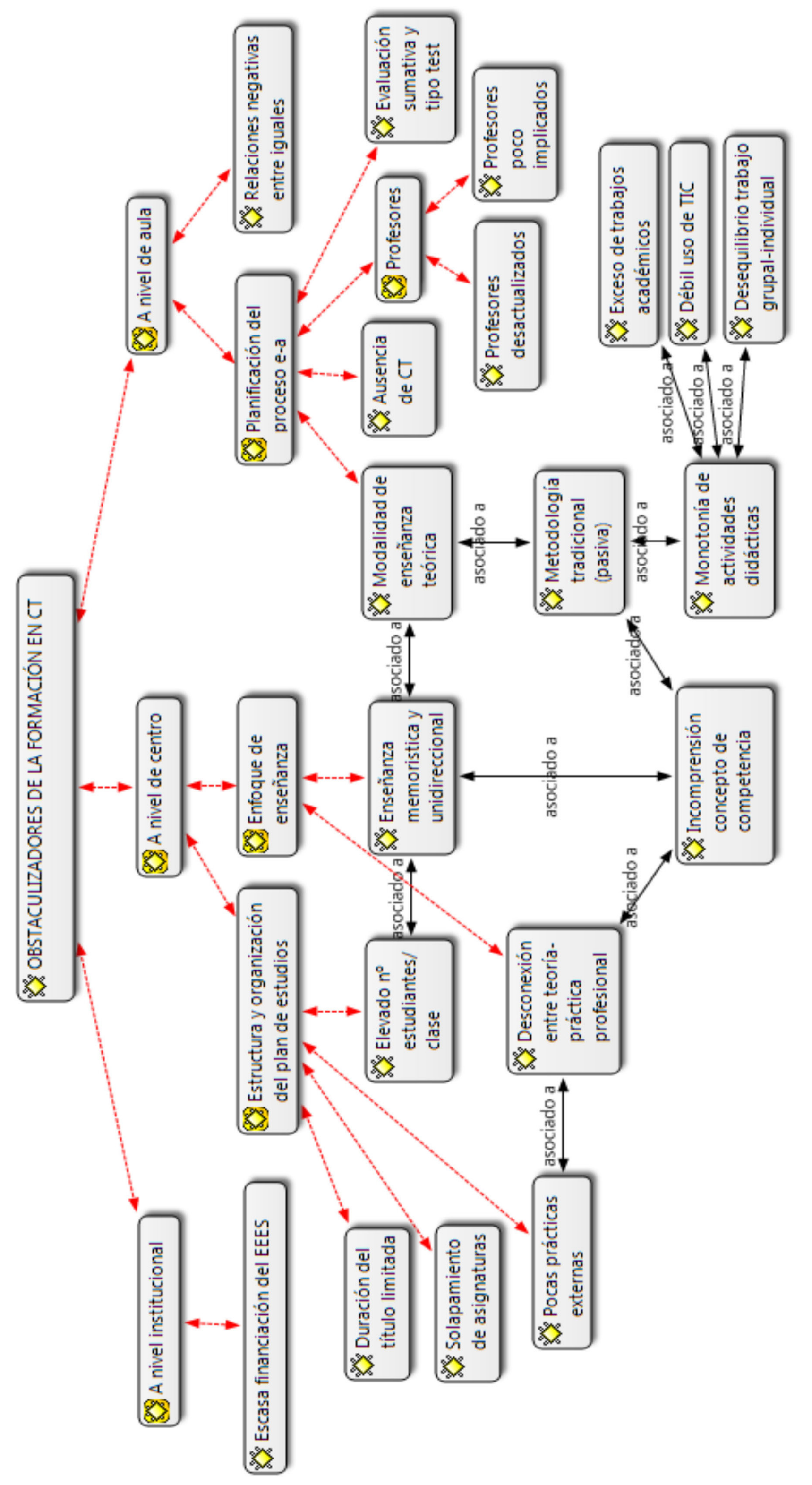

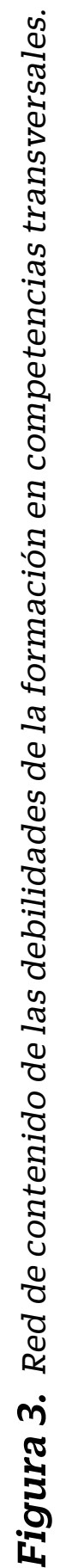


Los estudiantes reconocen solapamiento entre las asignaturas con contenidos repetidos e, incluso, asignaturas de relleno que no aportan mucho; una escasez de tiempo para desarrollar todas las competencias, además de una distribución inapropiada de horarios y asignaturas en los diferentes cursos, con poco tiempo y dedicación en algunas ocasiones a las competencias transversales y asignaturas que son fundamentales en la carrera (no miden el tiempo y nos dan mucha materia muy rápido).

Por otro lado, piensan que realizan pocas prácticas externas lo que les lleva a considerar una desconexión de la teoría o formación recibida con la práctica profesional. Las reducidas horas de prácticas externas es una de las principales limitaciones que encuentran los estudiantes para el desarrollo de las competencias transversales, extensible a todas las ramas de conocimiento. Las competencias se desarrollan en la acción, viviendo y experimentando y como tal, echan en falta el sentido práctico de la formación al considerar que tienen un plan de estudios desenfocado de la realidad (...), que no prepara (...) para problemas reales.

Esta limitación, junto con la percepción del estudiante sobre el elevado número de alumnos por clase, refleja la incomprensión del concepto de competencia en la formación universitaria con profesores que no entienden ni comparten este tipo de formación, siendo esta otra de sus obstaculizadores. Los estudiantes perciben demasiados alumnos en el aula que implica llevar a cabo el enfoque de enseñanza unidireccional y memorística, contraproducente en una formación basada en competencias. Este último código se comporta de nexo de unión con la tercera subcategoría que contempla las limitaciones a nivel de aula.

Dentro de los aspectos relacionados con la planificación del proceso de enseñanzaaprendizaje, la más recurrida por los estudiantes es la modalidad de enseñanza teórica, junto con una metodología tradicional basada en la lección magistral. Los alumnos consideran que las clases magistrales impiden fomentar su capacidad de investigar, crear y aprender de forma general, además de suponer un rollo (...) machacante que se convierte en un clavo que no te deja pensar.

Asociada a esta unidad de análisis se encuentra la percepción del estudiante sobre la monotonía de trabajos y actividades didácticas, siempre lo mismo y con poca creatividad por parte de los profesores. Se percibe un escaso uso de los materiales audiovisuales, además de los mismos trabajos año tras año, en los que predomina el trabajo en grupo. A este respecto, existe un exceso de trabajo en grupo que desestabiliza el peso con el trabajo autónomo, tan sugerido desde el EEES (abuso del trabajo cooperativo).

A pesar de que el paradigma educativo cambia, parece que los docentes siguen empleando su metodología tradicional y actividades alejadas de la filosofía formativa actual. Por ello, no es de extrañar que el alumnado perciba, por un lado, una ausencia generalizada de las competencias transversales en las aulas, apenas se nombran y se trabajan en clase y, por otro, profesores desactualizados y poco implicados en dicha formación (muchos docentes no saben cómo trabajarlas de forma adecuada). Este último aspecto ha sido ampliamente compartido 
por el alumnado, al que cabe añadir, el sistema o estrategias de evaluación empleadas. Se hace hincapié en un exclusivo interés por la nota del examen, sin herramientas apropiadas para la evaluación de dichas competencias. Según los estudiantes, se da poca importancia a su evaluación en las asignaturas, existe una falta de mecanismos para evaluar competencias y el examen tipo test (...) se considera el único instrumento de evaluación.

Por último, las relaciones negativas entre el alumnado, como la competitividad asociada a una falta de compañerismo, es otro de los aspectos que, según el propio estudiante, dificulta el desarrollo de las competencias transversales en las aulas universitarias.

\section{Propuestas de mejora para la formación en competencias transversales en la Educación Superior}

Realizada la codificación de las fortalezas y debilidades de la formación en competencias transversales, consistente en relacionar todos los códigos en torno a categorías centrales en busca de un línea narrativa-interpretativa en la cual todas las unidades de información están contempladas, el siguiente paso consiste en interpretar la categorización de las propuestas de mejora dadas por los estudiantes siguiendo la misma línea argumental.

En las propuestas de mejora convergen tres grandes subcategorías que agrupan las unidades de análisis (códigos) en función de su extensión (a nivel institucional, de centro o aula). Todas ellas recogidas, de forma general y común en todos los Grados analizados, en la red de contenido siguiente (ver Figura 4).

La primera subcategoría hace referencia a propuestas de mejora a nivel institucional. En este caso las propuestas más comunes van dirigidas fundamentalmente a incrementar la oferta de actividades extracurriculares en la universidad que posibilite al estudiante completar su formación en competencias transversales a través de cursos formativos, actividades culturales o sociales y voluntariados. De forma concreta los estudiantes proponen que la universidad ofrezca cursos específicos para la enseñanza de las competencias transversales y se enriquezcan de las experiencias de voluntariado o intercambios. Por otro lado, los estudiantes hacen hincapié en la importancia de potenciar la orientación profesional en todos los títulos de grado en dos sentidos: para dar a conocer las salidas profesionales explicando cómo funciona verdaderamente el mundo laboral y las opciones que tenemos una vez acabado el Grado y ayudar a planificar el futuro profesional del estudiante.

La segunda subcategoría contempla propuestas de mejora a nivel de facultad o centro. Esta subcategoría incluye diferentes familias categóricas que merecen una mención especial. 

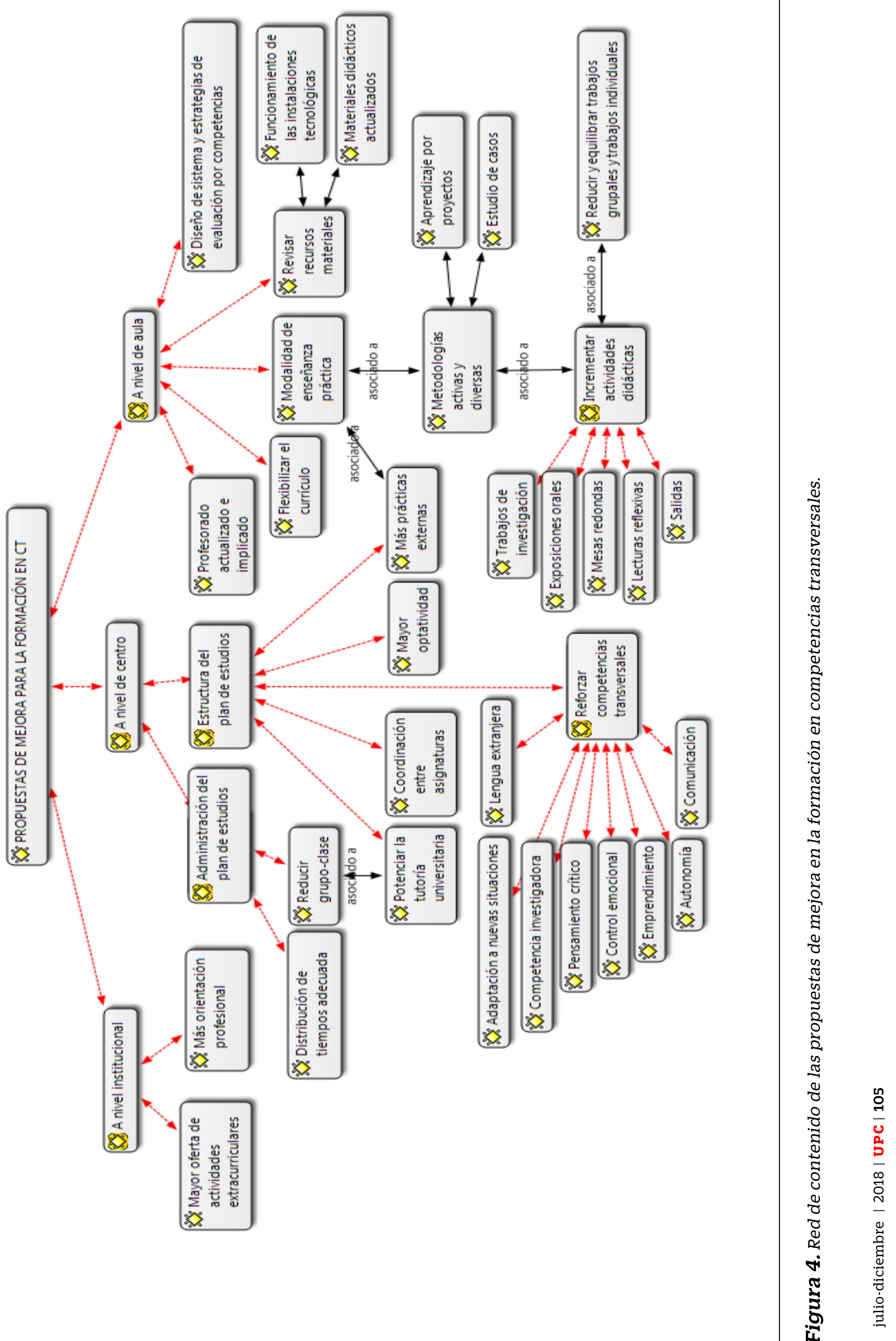
Es el caso de las propuestas que giran en torno a la administración del plan de estudios, como la revisión de la duración de los títulos y asignaturas para poder desarrollar todas estas competencias, y a la vez que las asignaturas con contenido especialmente relevante para la inserción del futuro profesional se le atribuyan más créditos en el currículo. Otra propuesta de este bloque es la reducción del número de alumnos por aula para obtener una enseñanza y atención más personalizada y acorde con los planteamientos de Bolonia (cumplir realmente lo que propone el Plan Bolonia y no hacer una cutre (pobre) interpretación de este). A esta afirmación se le añade el valor que el estudiante confiere a la tutoría universitaria para el desarrollo de estas competencias, planteando potenciarla tanto en un plano individual como grupal. Este código corresponde a la subcategoría denominada estructura del plan de estudios, con una función de enlace entre ambas.

La estructura del plan de estudios agrupa las propuestas referidas a potenciar las prácticas externas, aumentado la cantidad y duración de las mismas, ya que es ahí cuando aprendemos verdaderamente a interactuar (...) y a desarrollar nuestras competencias transversales, a la vez que permiten enfrentar al alumnado a la realidad social para que sepa actuar; además de desarrollar un currículo universitario con una oferta de optativas más amplia (buscaría una mayor oferta de prácticas y optativas adaptadas a los intereses del alumnado). Con ello el estudiante propone incluir asignaturas y contenidos concretos para el desarrollo de las competencias transversales, como por ejemplo dirigidas al conocimiento de una lengua extranjera. Asimismo, el estudiante señala también la necesidad de mejorar la coordinación entre asignaturas, promoviendo la colaboración, comunicación y cooperación entre los profesores de manera que todos los departamentos puedan trabajar en la misma dirección y de forma más eficiente. Igualmente, evitar, por un lado, el solapamiento de contenidos y, por otro, lagunas formativas, para que se pueda aprovechar el tiempo dedicado a cada asignatura y así reforzar otros menos desarrollados en las aulas como es el caso de los contenidos próximos a las competencias transversales. Concretamente los participantes señalan que hay que reforzar las competencias de adaptación a nuevas situaciones, la investigadora, el pensamiento crítico, el control emocional, el emprendimiento, la comunicación y la lengua extranjera.

Por último, a nivel de aula se señalan distintas propuestas de mejora. Gran parte de ellas incurren fundamentalmente en la formación del profesorado y su motivación para desarrollarlas a través de su actualización formativa continua y el reconocimiento de su valor en la formación del graduado.

Trabajar las competencias transversales es una forma de mejorar la formación tanto a nivel académico como profesional. Acercar al estudiante a su realidad laboral es de las propuestas más citadas por los participantes. Entre la forma más apropiada para hacerlo es enfocar la docencia más a la práctica, es decir, potenciar la modalidad de enseñanza práctica basada en metodologías activas e innovadoras como el estudio de casos y el aprendizaje por proyectos. 
También aluden a las estrategias de aprendizaje cooperativo, pero siempre desde el equilibrio con el trabajo autónomo y con significado y coherencia.

A veces perciben un exceso de trabajo que proponen reconducir hacia la realización de otras actividades como exposiciones orales, mesas redondas, lecturas comprendidas, salidas o excursiones e, incluso, trabajos de investigación. Todos ellos aportan una riqueza de perspectivas que flexibilizan el currículo y favorecen el desarrollo de las competencias transversales entre la diversidad del alumnado.

Para llevar a cabo esta diversidad de actividades didácticas es necesario que el docente se apoye en recursos materiales actualizados y en unas instalaciones tecnológicas en buenas condiciones.

Por último, los estudiantes añaden la necesidad de elaborar un sistema de evaluación por competencias basado en exámenes reflexivos más que memorísticos y en el diseño de herramientas concretas para evaluarlas. Además de tener el desarrollo de los aspectos personales y sociales del alumnado más en cuenta a la hora de poner la nota final.

\section{DISCUSIÓN}

Ante la relevancia de las competencias transversales en el mercado laboral, en este trabajo se ha pretendido conocer su desarrollo en las aulas de la Universidad de Murcia, a partir de la percepción de uno de sus principales protagonistas: el alumnado.

Comoseaprecia enelapartadoderesultados, el desarrollodelascompetencias transversales es susceptible de mejora con una valoración media en torno al valor de 3. Estos resultados son coherentes con los de otros estudios (Alonso, Fernández \& Nyssen, 2009; CEDEFOP, 2014; Clemente \& Escriba, 2013; De la Iglesia, 2011; Mir, 2008), que no solo fijan su atención en la percepción del alumnado, sino también en la de docentes y empleadores. En todos ellos se pone de relieve una formación universitaria centrada en el desarrollo de competencias específicas, mientras que las transversales pasan a un segundo plano, a pesar de contemplarse en las guías docentes de las asignaturas. No obstante, en el presente trabajo destaca la competencia de trabajo en equipo como la más desarrollada (sobre todo, por el alumnado de Ciencias Sociales) y la de control emocional como la que menos (aunque Ingeniería es la rama de conocimiento en la que más se potencia).

Paradójicamente, el alumnado percibe el trabajo en equipo como una debilidad de la formación en competencias transversales en las aulas de la Universidad de Murcia. Lo que nos lleva a pensar que no es suficiente con trabajar de forma cooperativa, si previamente no se enseña a realizar este tipo de trabajo. Miró (2010) afirma que el logro de las competencias transversales requiere de una formación previa, no siendo distintas de las demás en este aspecto. Para que el alumnado trabaje en equipo, no basta con agruparlos, "esto es poco efectivo en el 
mejor de los casos y a menudo, contraproducente" (p.3). Según la opinión de los estudiantes, su predominio es excesivo, incluso se abusa de esta forma de plantear la realización de trabajos y actividades didácticas, lo que trae como consecuencia un desequilibrio entre el trabajo grupal y el autónomo.

Se aprecia, por tanto, una incomprensión del concepto de competencia. Como señalan Solanes et al. (2008), aunque en el proceso formativo se procure la adquisición y desarrollo de estas, no siempre se lleva a cabo de manera explicativa, intencional y controlada, sobre todo, en relación con las más genéricas, como es el caso de las transversales. Su inclusión en el currículo requiere de planteamientos académicos planificados e integradores.

Esta falta de comprensión a la hora de abordar las competencias transversales en el aula se refleja también en los sistemas de evaluación establecidos por el profesorado, vistos como otra debilidad por el alumnado y por los resultados de las investigaciones de Blanco (2009) y Cobo y Moravec (2011). Según estos autores, difícilmente una evaluación en la que se valoran únicamente los conocimientos adquiridos puede reflejar la adquisición de competencias. En su defecto, estas requieren de sistemas más reflexivos y de acción donde se puedan demostrar, poner en práctica (Robledo et al., 2015).

En cuanto a las debilidades apuntadas por el alumnado con relación al centro, las principales apuntan a la estructura y organización del plan de estudios y cómo, al mantener un elevado número de estudiantes por clase, se propicia una desconexión entre teoría y práctica laboral, al predominar una enseñanza memorística y unidireccional, dejando de lado metodologías más innovadoras y coherentes con el principio de aprender haciendo, que plantea la convergencia europea.

Estas opiniones corroboran el trabajo de Michavila et al. (2016) quienes detectan un predominio excesivo de la clase magistral en la universidad, "poco adecuado para el paradigma educativo actual" (p.47), ya que minimiza las posibilidades de desarrollar un aprendizaje activo, dinámico y acorde con la actual formación basada en competencias en la que el aprendo, practico y aplico se convierten en premisas básicas, tal y como defienden Gargallo, Garfella, Sahuquillo, Verde y Jiménez (2015) y Robledo et al. (2015). Esta falta de innovación metodológica puede deberse, a su vez, a una falta de implicación y/o formación del docente con relación a la nueva concepción de la enseñanza basada en competencias. Esta debilidad también se pone de manifiesto en el trabajo de Martín (2009), que detecta cierta dificultad para incorporar a la cultura de los docentes esta visión actualizada de la formación universitaria.

Paralelamente, el alumnado también apunta como debilidad el poco contacto con la realidad de la profesión, considerados reducidos los créditos otorgados a las prácticas externas. Como apuntan Tejada y Navío (2005), el desarrollo de las competencias es indisociable de su aplicación o puesta en marcha, por lo que los estudiantes aluden al periodo de prácticas como uno de los escenarios más apropiados para el desarrollo de las competencias transversales, al 
igual que Freire et al. (2013) y Mir (2008) ponen de manifiesto con los resultados de sus trabajos.

Por último, el alumnado encuentra la escasa financiación recibida por las universidades una debilidad para implantar una reforma de semejante profundidad en el sistema universitario español. Michavila (2012) considera que el cambio que plantea la convergencia europea no se ha producido, tan solo a nivel conceptual y localiza en la financiación uno de los principales factores que obstaculizan la profundidad de los cambios que deben producirse.

Por otra parte, entre las fortalezas apuntadas por los estudiantes de la Universidad de Murcia para la formación en competencias transversales, destaca su propia dedicación y capacidades, así como ciertas actividades extracurriculares como acciones formativas, voluntariado y becas de movilidad. Estas últimas se relacionan con distintos tipos de experiencias: internacionales, con iguales y laborales, lo que resalta la importancia de la alternancia de contextos en el desarrollo de las competencias transversales, como concluyen los estudios de Accenture y Universia (2007) y Martínez Clares y González Morga (2018c).

Resulta novedoso el valor que le atribuye el estudiante a la tutoría universitaria para personalizar su proceso formativo y, en consecuencia, ofrecer una respuesta más ajustada a sus necesidades. Los beneficios de este proceso orientador, así como su implicación en la calidad de la formación universitaria, se ponen de manifiesto en múltiples trabajos, como el de Álvarez y Álvarez (2015), Lobato y Guerra (2014) o Perez Cusó, González Lorente, Martínez Juárez y González Morga (2017). La tutoría cobra especial relevancia en un enfoque de enseñanza-aprendizaje basado en competencias, en el que el docente adquiere un rol de acompañante para procurar la formación integral del alumnado. Es por ello que se sitúa muy cerca del desarrollo de competencias transversales y, por tanto, como un medio para consolidar el sistema formativo actual.

Los participantes en este trabajo también realizan propuestas que pueden ayudar en el desarrollo de las competencias transversales en la Universidad de Murcia. Este es el caso de incrementar la oferta de actividades extracurriculares, ya que su vinculación con la adquisición de competencias transversales es un hecho puesto de manifiesto en investigaciones como las de Carrasco (2012), Cobo y Moravec (2011) o Rodríguez (2015).

Potenciar la orientación profesional también se encuentra entre sus prioridades, pues una de sus principales finalidades es la de facilitar el desarrollo de competencias que promuevan la empleabilidad, así como la de preparar al estudiante para que sea capaz de gestionar su proyecto profesional y de vida. Esta propuesta coincide con las medidas planteadas por Eurydice (2014) para mejorar la formación inicial de los graduados.

Al igual que Martín y Bobb (2011), el alumnado resalta la distribución de créditos por asignaturas, dada la importancia para su perfil profesional, y la reducción del número de estudiantes por clase como necesarias en la implantación de un sistema formativo basado en competencias.

La tutoría universitaria vuelve a ponerse de manifiesto como espacio de fomento del crecimiento personal de los estudiantes, de su rendimiento académico y generador de 
comunidad y de relaciones interpersonales, como subrayan las investigaciones de Pérez Cusó et al. (2017) y Sanz (2012).

Con vistas a conseguir coherencia, seguimiento y control en el aprendizaje, los participantes proponen la máxima coordinación entre asignaturas, cuyas implicaciones positivas se señalan en trabajos como los de Rué y Lodeiro (2010) o Velasco, Rodríguez, Terrón y García (2012).

En contraposición a las debilidades apuntadas por el alumnado, este propone, por una parte, más presencia de prácticas externas en los planes de estudio. La dimensión práctica en el desarrollo de competencias tiene una importancia incuestionable, razón por la que se considera que los títulos de Grado deberían ampliar la cantidad y duración de las prácticas externas, así como flexibilizar la oferta de centros para favorecer una elección más acorde con los intereses profesionales de los participantes. Tejada y Navío (2005) resaltan la relevancia de este medio para la adquisición de competencias, al ser indisociables de su desarrollo.

Por otra parte, los estudiantes proponen un mayor empleo de una modalidad de enseñanza más práctica y metodologías activas de aprendizaje. Gil e Ibáñez (2013) valora positivamente estas metodologías para comprender mejor la teoría y afrontar y resolver situaciones prácticas y reales. En esta línea, Carrasco (2012, p. 228) afirma que “la adquisición de competencias por parte del estudiante exige poner en juego un conjunto coherente y coordinado de estrategias metodológicas, recurriendo a diferentes actividades y espacios formativos". Esta forma de trabajar aproxima el proceso de enseñanza-aprendizaje a las exigencias de la vida profesional.

Al hilo de la afirmación anterior, aparecen otras propuestas realizadas por los estudiantes como el incremento y la alternancia de actividades didácticas, la flexibilidad curricular, la revisión de los recursos materiales, la adecuación del sistema de evaluación y la actualización y el compromiso del profesorado. Con relación a este último aspecto, y de nuevo en palabras de Carrasco (2012, p. 225), "cualquier cambio de paradigma educativo requiere actualización docente e incentivar la innovación", considerando la formación del profesorado como una de las cuestiones principales sobre las que prestar atención, puesto que sobre él recae de forma directa la responsabilidad del desarrollo de competencias en el alumnado, y no solo las de carácter más específico.

Como puede apreciarse, disponer de la percepción del alumnado sirve de referencia para reflexionar sobre las acciones más eficaces en pro del desarrollo de competencias, para potenciar así la calidad del proceso formativo, aunque también es necesario el compromiso de toda la comunidad universitaria para favorecer una práctica en sintonía con el nuevo paradigma educativo y los requerimientos del mercado laboral (Hargreaves \& Fullan, 2014). Existe la urgencia de un nuevo cambio formativo, en la que desarrollar aquellas competencias transversales que permitan aprender y adaptarse a un mundo del empleo que cambia rápidamente será el reto decisivo de nuestro tiempo. 


\section{REFERENCIAS}

Accenture \& Universia (2007). Las competencias profesionales de los titulados. Contraste y diálogo UniversidadEmpresa. Madrid: Centro de Alto Rendimiento de Accenture y Universia. Recuperado de https://goo.gl/bc8Qr7 Aguado, D., González, A., Antúnez, M., \& Dios, T. (2017). Evaluación de Competencias Transversales en Universitarios. Propiedades Psicométricas Iniciales del Cuestionario de Competencias Transversales. REICE, Revista Iberoamericana sobre Calidad, Eficacia y Cambio en Educación, 15(2), 129-152. doi: https://doi.org/10.15366/ reice2017.15.2.007

Alonso, L., Fernández, C. J., \& Nyssen, J. M. (2009). El debate sobre las competencias: Una investigación cualitativa en torno a la educación superior y el mercado de trabajo en España. Madrid: ANECA. Recuperado de https://goo.gl/3keShP

Álvarez, M., \& Álvarez, J. (2015). La tutoría universitaria: Del modelo actual a un modelo integral. Revista Electrónica InteruniversitariadeFormaciónDelProfesorado,18(2),125-142.doi:http://dx.doi.org/10.6018/reifop.18.2.219671

Aubert, J., \& Gilbert, P. (2003). L'evaluation des competences. Sprimont, Bélgica: Mardaga.

Blanco, A. (Coord.) (2009). Desarrollo y evaluación de competencias en Educación Superior. Madrid: Narcea.

Carrasco, A. (2012). Desarrollo de competencias transversales en los estudios de ingeniería y espacio formativo de blended-learning en la enseñanza de segundas lenguas(Tesis doctoral). Recuperado de https://goo.gl/nxCnxK

Centro Europeo para el Desarrollo de la Formación Profesional [CEDEFOP] (2014). Desajuste de competencias: Más de lo que parece a simple vista. Nota informativa. Recuperado de https://goo.gl/NwBPTf

Clemente, J. S., \& Escribá C. (2013). Análisis de la percepción de las competencias genéricas adquiridas en la Universidad. Revista de Educación, 362, 535-561. doi: http://dx.doi.org/10.4438/1988-592X-RE-2013-362-241

Cobo, C., \& Moravec, J. (2011). Aprendizaje invisible. Hacia una nueva ecología de la educación. Barcelona: UBe. Recuperado de https://goo.gl/mAluzr

De la Iglesia, M. C. (2011). Adecuación del grado de desarrollo de la formación en competencias a la necesidad en el entorno laboral, según la opinión de los estudiantes. Revista Complutense de Educación, 22(1), 71-92. doi: http://dx.doi.org/10.5209/rev_ RCED.2011.v22.n1.4

Eurydice (2014). Modernización de la Educación Superior en Europa: Acceso, permanencia y empleabilidad 2014. Informe Eurydice. Luxemburgo: Oficina de Publicaciones de la Unión Europea. Recuperado de https://goo. $\mathrm{gl} / \mathrm{d} 4 \mathrm{vChT}$

Freire, M. J., Teijeiro, M. M., \& Pais, C. (2013). La adecuación entre las competencias adquiridas por los graduados y las requeridas por los empresarios. Revista de Educación, 362, 13-41. doi: http://dx.doi.org/10.4438/1988-592XRE-2011-362-151

Gargallo, B., Garfella, P. R., Sahuquillo, P. M. Verde, I., \& Jiménez. M. A. (2015). Métodos centrados en el aprendizaje, estrategias y enfoques de aprendizaje en estudiantes universitarios. Revista de Educación, 270, 229-254. doi: http://dx.doi.org/10.4438/1988-592X-RE-2015-370-304

Gil, P., \& Ibáñez, A. (2013). Percepción de utilidad y grado de satisfacción del alumnado de formación del profesorado con el Método del Caso. Aula Abierta, 41(3), 79-90. Recuperado de https://goo.gl/xjfcAe

González Morga, N. (2017). Un estudio de competencias transversales en la Universidad de Murcia (Tesis doctoral). Recuperado de https://digitum.um.es/xmlui/handle/10201/52943

González, V., \& González, R. M. (2008). Competencias genéricas y formación profesional: Un análisis desde la docencia universitaria. Revista iberoamericana de Educación, 47, 185-209. Recuperado de http://goo.gl/HCo9rq

Hargreaves, A., y Fullan, M. (2014). Capital profesional. Madrid: Morata.

Herrero, R., González, I., \& Marín, V. (2015). Formación centrada en competencias estudiantiles en educación superior. Revista de Ciencias Sociales, 21(4), 461- 478. Recuperado de http://goo.gl/ja166x

Ibañez, J. A. (2009). La fundamentación filosófica de los nuevos planes de estudios. En A. Medina, M. L. Sevillano, \& S. 
de la Torre (Coord.), Una universidad para el siglo XXI: EEES (pp. 17-19). Madrid: Universitas.

Jato, E., Cajide, J., Muñoz, M., \& García, B. (2016). La formación del profesorado universitario en competencias lifelong learning a partir de las demandas de empleadores y egresados. Revista de Investigación Educativa, 34(1), 69 85. doi: http://dx.doi.org/10.6018/rie.34.1.215341

Lobato, C., y Guerra, N. (2014). Las tutorías universitarias en el contexto europeo. Orientación y sociedad, 14(1), 1-22. Recuperado de http:// goo.gl/QW1hxD

López Gómez, E. (2016). En torno al concepto de competencia: Un análisis de fuentes. Profesorado. Revista de Currículum y Formación del Profesorado, 20(1), 311-322. Recuperdo de https://goo.gl/gNP5pt

Manzanares, M. A., \& Sánchez, J. (2012). La dimensión pedagógica de la evaluación por competencias y la promoción del desarrollo profesional en el estudiante universitario. Revista Iberoamericana de Evaluación Educativa, 5(1), 186-202. doi: http://dx.doi.org/10.15366/riee

Mareque, M., \& De Prada, E. (2018). Evaluación de las competencias profesionales a través de las prácticas externas: incidencia de la creatividad. Revista de Investigación Educativa, 36(1), 203-219. doi: http://dx.doi.org/10.6018/ rie.36.1.275651

Martín, E. (2009). Profesorado competente para formar alumnado competente: El reto del cambio docente. En J. I. Pozo \& M. P. Echeverría (Eds.), Psicología del aprendizaje universitario: La formación en competencias (pp. 199215). Madrid: Morata

Martín, P., \& Bobb, L. (2011). La implantación de los nuevos grados: Propuestas de mejora. Revista de Educación, 356, 703-715. doi: http://dx.doi.org/10-4438/1988-592X-RE-2010-356-120

Martínez Clares, P. \& González Morga, N. (2018a). Las competencias transversales en la universidad: propiedades psicométricas de un cuestionario. Educación XX1, 21(1), 231-262, doi: http://dx.doi.org/10.5944/educXX1.15662

Martínez Clares, P., \& González Morga, N. (2018b). Metodologías de enseñanza en la universidad y su relación con el desarrollo de competencias transversales. Cultura y Educación, 30(2), 233-275. doi. https://doi.org/10.1080/11 356405.2018.1457610

Martínez Clares, P., \& González Morga, N. (2018c). El dominio de competencias transversales en Educación Superior en diferentes contextos formativos. Educacao e Pesquisa, 45. Recuperado de http://www.educacaoepesquisa. fe.usp.br/?cat=16

Michavila, F. (2012). Bolonia en crisis. Madrid: Tecnos.

Michavila, F., Martínez, J. M., Martín, M., García, F. J., y Cruz, J. (2016). Barómetro de empleabilidad y empleo de los universitarios en España, 2015 (Primer informe de resultados). Madrid: Observatorio de Empleabilidad y Empleo Universitarios. Recuperado de http://goo.gl/q7Ao7W

Mir, A. (2008). Las competencias transversales en la Universidad Pompeu Fabra. La visión de los docentes y estudiantes de segundo ciclo. Revista de Docencia Universitaria [REDU], 1, 1-16. Recuperado de http://revistas.um.es/redu/ article/view/10641

Miró, J. (2010). Para qué sirven las competencias transversales. Recuperado de http://bioinfo.uib.es/rjoemiro/CTens/ PorqueCT.pdf

OCDE (2016). Global Competency for an Inclusive World. Paris: OECD. Recuperado de http://goo.gl/TUqfXF

Organización Internacional del Trabajo [OIT] (2015). Tendencias mundiales del empleo juvenil 2015. Promover la inversión en empleos decentes para los Jóvenes. Ginebra: Autor. Recuperado de https://goo.gl/JITvIS

Palmer, A., Montaño, J., \& Palou, M. (2009). Las competencias genéricas en la educación superior: Estudio comparativo entre la opinión de empleadores y académicos. Psicothema, 21(3), 433-438. Recuperado de http://goo.gl/KszETX

Pérez Cusó, J., González Lorente, J., González Morga, N., \& Martínez Júarez, M. (2017). Tutoría en la Universidad: un 
estudio de caso en la Facultad de Educación de la Universidad de Murcia. Educatio siglo XXI, 35(2), 91-110. doi. https://doi.org/10.6018/j/298531

Robledo, P., Fidalgo, R., Arias, O., \& Álvarez, M.L. (2015). Percepción de los estudiantes sobre el desarrollo de competencias a través de diferentes metodologías activas. Revista de Investigación Educativa, 33(2), 369-383. doi: http:// dx.doi.org/10.6018/rie.33.2.201381

Rodríguez, R. M. (2014). Modelo formativo en el Espacio Europeo de Educación Superior: valoraciones de los estudiantes. Aula Abierta, 42(2), 106-113. http://doi.org/10.1016/j.aula.2014.03.002

Rodríguez, R. M. (2015). Competencias genéricas en la enseñanza superior a través de los programas de internacionalización. Revista Complutense de Educación, 26(1), 81-100. doi: http://doi.org/10.5209/ revRCED.2015.v26.n1.42598

Rodríguez, G., Ibarra, M. S., \& Cubero, J. (2018). Competencias básicas relacionadas con la evaluación. Un estudio sobre la percepción de los estudiantes universitarios. Educación XX1, 21(1), 181-208, doi: http://doi.org/10.5944/ educXX1.14457

Rué, J., \& Lodeiro, L. (2010). Equipos docentes y nuevas identidades académicas en educación superior. Madrid: Narcea Universitaria.

Rychen, D. S., \& Salganik, L. H. (Eds.) (2003). Key competencies for a successful life and well-functioning society. Gotinga: Hogrefe \& Huber Publishers.

Sanz, M. T. (2012). Estudio de la acción tutorial como paso previo a la implantación del EEES en la licenciatura de Administración y Dirección de Empresas de la Universidad de Huelva. Revista de Investigación Educativa, 3O(1), 145-160. doi http://dx.doi.org/10.6018/rie.30.1.115141

Sarceda, M.C., \& Rodicio, M.L. (2018). Escenarios formativos y competencias profesionales en la formación inicial del profesorado. Revista Complutense de Educación, 29(1) 2018: 147-164. doi: https://doi.org/10.5209/RCED.52160

Solanes, A., Núñez, R., \& Rodríguez, J. (2008). Elaboración de un cuestionario para la evaluación de competencias genéricas en estudiantes universitarios. Apuntes de Psicología, 26(1), 35-49. Recuperado de https://goo.gl/6quS1L

Teichler, U. (2015). Changing perspectives: The professional relevance of higher education on the way towards the highly-educated society. European Journal of Education, 50(4),1465-3435. doi: http://doi.org/10.1111/ejed.12146

Tejada, J. \& Navío, A. (2005). El desarrollo y la gestión de competencias profesionales: una mirada desde la formación. Revista Iberamericana de Educación, 37(2). Recuperado de https://rieoei.org/RIE/article/view/2719

United Nations Educational, Scientific and Cultural Organization [Unesco] (2016). 2015 Regional Study on Transversal Competencies in Education Policy and Practice (Phase III). Asia-Pacific Education Research Institutes Network (ERI-NET). París y Bangkok: Autor. Recuperado de https://goo.gl/BmqZ6r

Ursin, J. (2017). Transforming finnish higher education institutional mergers and conflicting academic identities. Revista de Investigación Educativa, 35(2), 307-316. doi. http://dx.doi.org/10.6018/rie.35.2.295831

Velasco, P., Rodríguez, R., Terrón, M. J., \& García, M. J. (2012). La coordinación del profesorado universitario: Un elemento clave para la evaluación por competencias. Revista de Docencia Universitaria [REDU], 1O(3), 265-284. doi https://doi.org/10.4995/redu.2012.6023

RIDU / Revista Digital de Investigación en Docencia Universitaria / ISNN 2223-2516

(C) Los autores. Este artículo es publicado por la Revista Digital de Investigación en Docencia Universitaria del Área de Institutional Research and Effectiveness de la Dirección de Aseguramiento de la Calidad, Universidad Peruana de Ciencias Aplicadas. Este es un artículo de acceso abierto, distribuido bajo los términos de la LicenciaCreativeCommons Atribución-CompartirIgual 4.0 Internacional ( http://creativecommons.org/licenses/by-sa/4.0/), que permite el uso no comercial, distribución y reproducción en cualquier medio, siempre que la obra original sea debidamente citada. 